\title{
Ability to access community-based needle-syringe programs and injecting behaviors among drug users: a cross-sectional study in Hunan Province, China
}

Lei Zhang ${ }^{1 *+}$, Xi Chen ${ }^{2+}$, Jun Zheng ${ }^{2}$, Junshi Zhao ${ }^{2}$, Jun Jing ${ }^{3^{*}}$, Jun Zhang ${ }^{3}$, Eric PF Chow ${ }^{1}$ and David P Wilson ${ }^{1}$

\begin{abstract}
Background: Needle-syringe exchange programs (NSPS) have been substantially rolled-out in China since 2002. Limited studies reported effectiveness of NSPs in a Chinese setting. This study aimed to assess the association between accessibility to NSPs and drug-use risk behaviors of IDUs by investigating primary (self-reported) data of IDUs recruited from NSP sites, community settings and mandatory detoxification centers (MDCs) in Hunan province, China.

Methods: A cross-sectional survey was conducted in Hunan province in 2010. IDU recruits participated in a face-to-face interview to provide information related to their ability to access NSPs, demographic characteristics, and injecting behaviors in the past 30 days.

Results: Of the total 402 participants, 35\%, 14\% and 51\% participants indicated low, medium and high ability to access NSPs in the past 30 days, respectively. A significantly higher proportion of IDUs (77.3\%) from the high-access group reported $\leq 2$ injecting episodes per day compared with medium- (46.3\%) and low-access (58.8\%) groups. Only $29.0 \%$ of high-access IDUs re-used syringes before disposal in the past 30 days, significantly lower than those in the medium- (43.1\%) and low-access (41.3\%) groups. Reported levels of needle/syringe sharing decreased significantly as the ability to access NSPs increased $(16.3 \%, 12.7 \%$ and $2.5 \%$ in the low, medium and high access groups, respectively). Ninety percent of IDUs recruited from MDCs had low ability to access NSPS.
\end{abstract}

Conclusions: Increased NSP accessibility is associated with decreased levels of injecting frequency, repetitive use and sharing of injecting equipment among Chinese IDUs. Mandatory detention of IDUs remains as a major barrier for IDUs to access NSPs in China.

\section{Introduction}

HIV epidemics usually first emerge in China among people who inject drugs. HIV prevalence among injecting drug users (IDUs) in southwest and northwest China has stabilized at $\sim 20 \%$ in the past decade [1-4], with infection spreading across to in other at-risk populations and the general population [5,6]. By 2009, 32.2\% of the estimated 740,000 people living with HIV in China [7] were infected

\footnotetext{
* Correspondence: Izhang@kirby.unsw.edu.au; jingjun@tsinghua.edu.cn ${ }^{\dagger}$ Equal contributors

${ }^{1}$ The Kirby Institute, The University of New South Wales, Sydney, NSW, Australia

${ }^{3}$ Comprehensive AIDS Research Center, Tsinghua University, Beijing, China Full list of author information is available at the end of the article
}

through sharing of injecting equipment [1]. Needle-syringe exchange programs (NSPs) have been progressively rolledout in major Chinese cities since 2002 [8]. By 2010, a total of 1023 NSPs sites have been established across 453 Chinese counties [9]. Access by IDUs to NSPs is largely associated with awareness of the programs, extent of police presence and risk of incarceration [10]. Illicit drug use is punishable by law in China. Upon arrest, IDUs are often directed to methadone maintenance therapy (MMT) clinics under supervision of the police. If relapse occurs during treatment, they are transferred to mandatory detoxification centers (MDCs) or labor camps for a period of between one and three years for compulsory drug rehabilitation [11-13]. In these institutions,

\section{Biomed Central}


access to harm reduction services is essentially nonexistent. Currently, institution-based interventions, including mandatory detoxification, voluntary rehabilitation and MMT, cover only $15 \%$ of the total drug users population [14], indicating a large gap for the scale-up of communitybased interventions, such as NSPs.

NSPs have been shown to be a safe and effective means to reduce syringe sharing and subsequent HIV transmission among IDUs in various settings [15-20]. Although NSPs have been implemented for almost a decade in China, only limited studies evaluate the effectiveness of NSPs in alleviating risk behaviors among Chinese IDUs and all have been conducted in high-HIV transmission provinces (Sichuan [21], Guangxi [21,22] and Guangdong [22,23]). Based on a cross-sectional study design, this study further investigates (1) the association between the ability to access NSPs and injecting behaviors in a low-HIV transmission setting; and (2) the impact of mandatory detention on IDUs' ability to access NSPs.

\section{Methods}

Study site, design and population

Hunan province is located in South China, bordered by Guangdong and Guangxi provinces to the south and Guizhou province to the west. All three adjacent provinces are traditional drug-trafficking provinces with exceptionally high HIV prevalence levels among IDUs [1]. Hunan's location has led to its role of channeling illicit drugs to other Chinese provinces [24]. A cross-sectional survey was conducted in three cities (Hengyang, Yiyang and Huaihua) of Hunan province from September to October 2010. Study participants were recruited from the community, NSP sites and MDCs. Community IDUs were recruited by outreach and peer-referral, whereas IDUs from NSP sites and MDC were recruited at venues. In MDCs, only IDUs admitted recently ( $<3$ months) were recruited to recall their injecting behaviors 30 days before their incarceration. Recent MDC entrants were particularly chosen to reduce recall bias.

Upon informed consent, participants completed a 30-minute questionnaire through face-to-face interviews; no identifying personal information was collected. The standardized questionnaire included questions related to demographic characteristics and injecting behaviors in the last 30 days (Table 1). In particular, the participants' ability to access NSPs was measured as the proportion of needle/ syringes obtained from NSPs in the past three months. For incarcerated drug users, this refers to their ability to access before incarceration.

\section{Statistical analysis}

Questionnaire data were double-entered and checked in EpiData (v3.0). For analyses, study participants were
Table 1 Demographic Characteristics, NSP accessibility and diseases prevalence levels among recruited IDUs

\begin{tabular}{|c|c|c|}
\hline Characteristics & $\mathbf{N}$ & Percent (\%) \\
\hline \multicolumn{3}{|l|}{ Venue of recruitment $(n=402)$} \\
\hline Needle and syringe program & 153 & 38.1 \\
\hline Mandatory Detoxification Centers & 78 & 19.4 \\
\hline Community & 171 & 42.5 \\
\hline \multicolumn{3}{|l|}{ Gender $(n=402)$} \\
\hline Male & 333 & 82.8 \\
\hline Female & 69 & 17.2 \\
\hline \multicolumn{3}{|l|}{ Age $(n=402)$} \\
\hline$<20$ & 4 & 1 \\
\hline $20-29$ & 98 & 24.4 \\
\hline $30-39$ & 180 & 44.8 \\
\hline $40-49$ & 116 & 28.9 \\
\hline$\geq 50$ & 4 & 1 \\
\hline Average $( \pm S D)$ & & $7 \pm 7.05$ \\
\hline \multicolumn{3}{|l|}{ Ethnic $(n=400)$} \\
\hline Han & 394 & 98.5 \\
\hline Non-Han & 6 & 1.5 \\
\hline \multicolumn{3}{|l|}{ Education $(n=402)$} \\
\hline Primary or below & 86 & 21.4 \\
\hline Junior high school & 252 & 62.7 \\
\hline Secondary high school & 54 & 13.4 \\
\hline College or above & 10 & 2.5 \\
\hline \multicolumn{3}{|l|}{ Marital Status ( $n=402)$} \\
\hline Single & 166 & 41.3 \\
\hline Married and cohabited & 139 & 34.6 \\
\hline Divorced and widowed & 97 & 24.1 \\
\hline \multicolumn{3}{|l|}{ Currently employed ( $n=395)$} \\
\hline Yes & 30 & 7.5 \\
\hline No & 365 & 92.5 \\
\hline \multicolumn{3}{|c|}{ Respondents' accessibility to NSP $(n=402)$} \\
\hline High (>70\%) & 142 & 35.3 \\
\hline Medium (40-70\%) & 55 & 13.7 \\
\hline Low (<40\%) & 205 & 51.0 \\
\hline
\end{tabular}

stratified into groups according to (1) their ability to access NSPs (i.e. low-access $[<40 \%]$, medium-access [40-70\%] and high-access groups [>70\%]) and (2) their venue of recruitment (Table 2). Descriptive statistics were calculated in the Statistical Package for the Social Sciences (v19) for Windows. Chi-square tests were used to assess differences between groups.

\section{Ethical considerations}

Ethics approval was obtained from the Institutional Review Board of the Tsinghua University (Project Code: 
Table 2 Injecting and sharing behaviors among 402 recruited IDUs

\begin{tabular}{|c|c|c|c|c|c|c|c|c|c|}
\hline & \multirow[t]{2}{*}{ Overall } & \multicolumn{3}{|c|}{ Ability to access to NSP syringes ${ }^{\ddagger}$} & \multirow{3}{*}{$\begin{array}{c}x^{2} \text {-test } \\
\left(x^{2}, p \text {-value }\right)\end{array}$} & \multicolumn{3}{|c|}{ Venue stratification } & \multirow{3}{*}{$\begin{array}{c}x^{2} \text {-test } \\
\left(x^{2}, p \text { value }\right)\end{array}$} \\
\hline & & $<40 \%$ & $40-70 \%$ & $>70 \%$ & & MDC & Community & NSP & \\
\hline & $\mathrm{N}(\%)$ & $\mathrm{N}(\%)$ & $\mathrm{N}(\%)$ & $\mathrm{N}(\%)$ & & $\mathrm{N}(\%)$ & $\mathrm{N}(\%)$ & $\mathrm{N}(\%)$ & \\
\hline \multicolumn{10}{|c|}{ Used drugs in the last 30 days $(n=402)^{*}$} \\
\hline Yes & $398(99.0)$ & $139(97.9)$ & $55(100)$ & $204(99.5)$ & & $74(94.9)$ & $171(100)$ & $153(100)$ & \\
\hline No & $4(1.0)$ & $3(2.1)$ & $0(0)$ & $1(0.5)$ & $2.89, p=0.236$ & $4(5.1)$ & $0(0)$ & $0(0)$ & $16.782, p<0.001+$ \\
\hline \multicolumn{10}{|c|}{ Frequency of injecting drugs in the last 30 days $(n=392)$} \\
\hline$<1$ times / day & $41(10.5)$ & $12(8.8)$ & $4(7.4)$ & $25(12.4)$ & & $7(9.6)$ & $19(11.1)$ & $15(10.1)$ & \\
\hline 1-2 times / day & $220(56.1)$ & $68(50.0)$ & $21(38.9)$ & $131(64.9)$ & & $37(50.7)$ & $109(63.7)$ & $74(50.0)$ & \\
\hline 3-5 times / day & $115(29.3)$ & $46(33.8)$ & $28(51.9)$ & $41(20.3)$ & & $22(30.1)$ & 39 (22.8) & $54(36.5)$ & \\
\hline$>6$ times / day & $16(4.1)$ & $10(7.4)$ & $1(1.9)$ & $5(2.5)$ & $29.439, p<0.001 \dagger$ & $7(9.6)$ & $4(2.3)$ & $5(3.4)$ & $15.236, p=0.019+$ \\
\hline
\end{tabular}

Times of repeated use per needle/syringe before disposal in the last 30 days $(n=389)$

\begin{tabular}{|c|c|c|c|c|c|c|c|c|c|}
\hline 0 & $4(1.0)$ & $0(0)$ & $3(5.9)$ & $1(0.5)$ & & $0(0)$ & $0(0)$ & $4(2.7)$ & \\
\hline 1 & $248(63.8)$ & 81 (58.7) & $26(51.0)$ & $141(70.5)$ & & $49(66.2)$ & $111(65.7)$ & $88(60.3)$ & \\
\hline $2-5$ & $131(33.7)$ & 54 (39.1) & $22(43.1)$ & $55(27.5)$ & & $21(28.4)$ & $57(33.7)$ & $53(36.3)$ & \\
\hline $6-9$ & $1(0.3)$ & $1(0.7)$ & $0(0)$ & $0(0)$ & & $1(1.4)$ & $0(0)$ & $0(0)$ & \\
\hline$\geq 10$ & $5(1.3)$ & $2(1.4)$ & $0(0)$ & $3(1.5)$ & $6.84, p=0.033 t$ & $3(4.1)$ & $1(0.6)$ & $1(0.7)$ & $0.151, p=0.927$ \\
\hline verage $\pm S D$ & $1.62 \pm 1.32$ & $1.80 \pm 1.45$ & $1.55 \pm 0.86$ & $1.52 \pm 1.31$ & & $1.93 \pm 2.00$ & $1.54 \pm 1.05$ & $1.56 \pm 1.13$ & \\
\hline
\end{tabular}

Shared syringes in the last 30 days $(n=400)$

\begin{tabular}{|c|c|c|c|c|c|c|c|c|c|}
\hline Yes & $35(8.8)$ & $23(16.3)$ & 7 (12.7) & $5(2.5)$ & & 15 (19.5) & $4(2.3)$ & $16(10.5)$ & \\
\hline No & $365(91.2)$ & $118(83.7)$ & $48(87.3)$ & $199(97.5)$ & 21.326, $p<0.001 \dagger$ & $62(80.5)$ & $167(97.7)$ & $136(89.5)$ & $20.507, p<0.001 \dagger$ \\
\hline
\end{tabular}

Number of times shared syringes in the last 30 days $(n=29)$

\begin{tabular}{|c|c|c|c|c|c|c|c|c|c|}
\hline 1 & $10(34.5)$ & $9(45.0)$ & $1(16.7)$ & $0(0)$ & & $8(66.7)$ & $0(0)$ & $2(15.4)$ & \\
\hline $2-5$ & $16(55.2)$ & $9(45.0)$ & $5(83.3)$ & $2(66.7)$ & & $4(33.3)$ & $3(75.0)$ & $9(69.2)$ & \\
\hline$\geq 6$ & $3(10.3)$ & $2(10.0)$ & $0(0)$ & $1(33.3)$ & $4.83, p=0.090$ & $0(0)$ & $1(25.0)$ & $2(15.4)$ & $5.780, p=0.056$ \\
\hline Average $\pm S D$ & $4.28 \pm 6.85$ & $4.15 \pm 7.37$ & $2.17 \pm 0.98$ & $9.33 \pm 9.29$ & & $1.55 \pm 0.93$ & $6.75 \pm 8.85$ & $5.92 \pm 8.78$ & \\
\hline \multicolumn{10}{|c|}{ Number of sharing partners in the last 30 days $(n=31)$} \\
\hline 1 & $10(31.3)$ & $5(22.7)$ & $5(71.4)$ & $0(0)$ & & $4(28.6)$ & $1(25.0)$ & $5(35.7)$ & \\
\hline $2-5$ & $20(62.5)$ & $15(68.3)$ & $2(28.6)$ & $3(100)$ & & $9(64.3)$ & $3(75.0)$ & $8(57.1)$ & \\
\hline $6-9$ & $1(3.1)$ & $1(4.5)$ & $0(0)$ & $0(0)$ & & $0(0)$ & $0(0)$ & $1(7.1)$ & \\
\hline$\geq 10$ & $1(3.1)$ & $1(4.5)$ & $0(0)$ & $0(0)$ & $6.73, p=0.035 \dagger$ & $1(7.1 \%)$ & $0(0)$ & $0(0)$ & $0.594, p=0.743$ \\
\hline Average $\pm S D$ & $2.53 \pm 1.98$ & $2.82 \pm 2.26$ & $1.43 \pm 0.79$ & $3.00 \pm 0.0$ & & $2.64 \pm 2.50$ & $2.25 \pm 0.96$ & $2.85 \pm 1.99$ & \\
\hline \multicolumn{10}{|c|}{ Maximum number of group sharing partners in the last 30 days $(n=32)$} \\
\hline 1 & $2(6.3)$ & $1(4.5)$ & $1(14.3)$ & $0(0)$ & & $1(7.1)$ & $0(0)$ & $1(7.1)$ & \\
\hline $2-5$ & $28(87.5)$ & $20(90.9)$ & $5(71.4)$ & $3(100)$ & & $13(92.9)$ & $4(100)$ & $11(78.6)$ & \\
\hline $6-9$ & $2(6.3)$ & $1(4.5)$ & $1(14.3)$ & $0(0)$ & $3.19, p=0.203$ & $0(0)$ & $0(0)$ & $2(14.3)$ & $0.867, p=0.648$ \\
\hline Average $\pm S D$ & $2.94 \pm 1.68$ & $2.86 \pm 1.42$ & $3.00 \pm 2.71$ & $3.33 \pm 0.58$ & & $2.50 \pm 0.76$ & $2.50 \pm 0.58$ & $3.50 \pm 2.35$ & \\
\hline
\end{tabular}

₹ Ability to access to NSP syringes is defined as the proportion of syringes obtained from NSPs during the past 3 months at the time of survey. $\dagger \mathrm{p}$-value is significantly $(<0.05)$ by Chi- 2 test.

* Number in bracket refers to the number of participants who answered the particular question.

0020120508). The survey was collected confidentially and anonymous with no names and personal information obtained. Verbal and written consent procedures were provided to all participants before the survey, and they had the right to withdraw from the study at any time without penalty.

\section{Results}

\section{Characteristics of study participants}

A total of 402 IDUs (community 42.5\%; NSP sites $38.1 \%$; MDC 19.4\%) participated in this study. Of all participants, 333 were male and 69 were female. Ages ranged between 17 to 56 years (mean $34.6 \pm 7.1$ years). The majority of 
respondents was ethnically Han (98.5\%), had finished junior high school (84.1\%), was currently unemployed $(92.5 \%)$ and was single/divorced (65.4\%) (Table 1). About 35\%, 14\% and $51 \%$ of participants reported low, medium and high ability to access NSPs in the past three months. Ninety percent $(90 \%)$ of IDUs recruited from MDCs had low ability to access NSPs, much higher than recruits from community $(24.6 \%)$ and NSP sites (19.0\%). In contrast, $62.0 \%$ and $61.4 \%$ of community and NSP recruits obtained $>70 \%$ of their syringes from NSPs, whereas only $6.3 \%$ of recent MDC entrants obtained this level of their syringes from NSPs.

\section{Injection risk behaviors}

Almost all (99\%) IDUs injected and consumed heroin exclusively (97.0\%) in the past 30 days. A significantly higher proportion of IDUs in the high-access group (77.3\%) reported no more than two injecting episodes per day compared with low (58.8\%) and medium-access groups $(46.3 \%)\left(x^{2}=29.44, p<0.001\right)$. When stratified by recruitment venues, the proportion (74.8\%) of IDUs reporting no more than two injecting episodes per day is significantly higher among community IDUs than those from NSP sites $(60.1 \%)$ and MDCs $(60.3 \%)\left(x^{2}=15.24\right.$, $\mathrm{p}=0.019)$. Only $29.0 \%$ of high-access IDUs reported having re-used syringes before disposal in the past 30 days, significantly lower than the medium and lowaccess groups $\left(43.1 \%\right.$ and $41.3 \%$ respectively) $\left(\chi^{2}=6.84\right.$, $\mathrm{p}=0.033$ ). Pattern of repeated use of syringes did not vary substantially across recruitment venues (Table 2).

Rates of needle/syringe sharing decreased substantially as ability to access NSPs increased $(16.3 \%, 12.7 \%$ and $2.5 \%$ in the low-, medium- and high-access groups respectively). Respondents from MDCs had the highest syringe sharing rate (19.5\%) 30 days prior to their incarceration in comparison with recruits from community $(2.3 \%)$ and NSP sites $(10.5 \%) \quad\left(x^{2}=20.51, p<0.001\right)$. Among those who shared syringes, an IDU shared on an average of $4.28 \pm 6.85$ occasions with $2.53 \pm 1.98$ sharing partners. The average sharing group size is approximately three (Table 2).

\section{Discussion}

Consistent with previous findings in both international [25-30] and Chinese settings [21-23], our findings indicate that provision of clean needle/syringes does not increase the injecting frequency among IDUs, and furthermore that increased availability of clean needles/ syringes may substantially contribute to the reduction in repeated use of dull syringes and sharing activities among Chinese IDUs. Liu et.al., in 2007, demonstrate that the rate of syringe sharing in the past 30 days among IDUs who regularly attend NSPs (15\%) is two to three times lower than non-attendees (32-44\%) in
Guangxi and Sichuan provinces [21]. Wu et.al., through a 12-month cohort study, show that the sharing rate dropped from $68 \%$ to $35 \%$ in the NSP intervention community in South China. Although, in comparison, IDUs in Hunan have a much lower sharing rate in general (low-access group: 16.3\%), an increased ability to access NSPs is associated with substantial reductions in sharing behaviors (high-access group: $2.5 \%$ ).

IDU recruits from MDCs exhibit significantly higher sharing rates, indicating a higher level of risk behaviors. Lower ability to access NSPs prior to their incarceration also suggests that the participation in MMT and likely police supervision may have prevented IDUs from accessing NSPs. In contrast, approximately two-thirds of the community and NSP recruits obtained more than $80 \%$ of their syringes from NSPs, suggesting NSPs are generally available and accessible to the majority of IDUs outside of the detention settings. In addition, confinement in MDCs does not reduce drug use behaviors [31-33] and as high as 95\% of IDUs relapse for drug addiction within one year of release [34]. Detainees also have limited access to health information and education that are often associated with NSPs. Together with our findings, this indicates that mandatory detoxification may be a major obstacle for harm reduction programs and improving risk behaviors for Chinese IDUs.

The first major limitation of this study is its crosssectional design. This does not enable us to directly investigate the causal relationship between NSP accessibility and its direct impact on drug use behaviors, although it provides an association analysis between the two. Second, the ability to access NSPs is defined as the self-reported percentage of acquired NSPs syringes in the past three months, which may be subjected to selfrecall bias. Third, although the study has been carefully designed to obtain information of injecting behaviors of recent MDC entrants prior to their admittance, the information may underestimate their actual risk behaviors due to prior harm reduction programs (e.g. MMT) $[35,36]$ and the temporary abstinence of injecting behaviors during the transferring process to MDCs by the police.

Our study has clearly demonstrated behavioral improvements when the ability to access NSPs increases in China. In addition, NSPs have been shown to be highly cost-effective in both international [37-40] and Chinese settings [41]. Full scale roll-out of NSPs should be implemented as a major component of harm reduction strategies nationwide. However, continued law enforcement and mandatory detoxification remain as major barriers to the necessary program scale-up and may even counteract the benefits of NSPs [11,42]. Ongoing police crackdowns, arrests and confinement substantially discourage IDUs from contacting peer health educators and 
accessing NSP sites. In comparison, in places where police are supportive of NSPs, coverage of the programs quickly increased and risk behaviors decreased [43]. Without effective cooperation between legislation, law enforcement and health policy sectors, NSPs are unlikely to reach a sufficient proportion of IDUs to make a significant impact on China's HIV epidemic.

\section{Competing interests}

The authors declare that they have no competing interests.

\section{Authors' contributions}

EPFC and LZ performed data analyses and wrote the Method and Result sections of the manuscript. XC, JZ1, JSZ conducted the field study and data collection. JZ2 and JJ participated in study design. LZ and XC wrote the manuscript. DPW assisted with data analyses and was responsible for the supervision of the project. All authors read and approved the final version of the manuscript.

\section{Funding source}

The study was supported by the following grants: the Australian Government Department of Health and Ageing, the University of New South Wales, the Endeavour Research Fellowship (Award ID: 2744_2012), the World Bank Global HIV/AIDS Program, and the Australian Research Council (Grant No.: FT0991990); Vice-Chancellor Fellowship, University of New South Wales, 2009-2012.

\section{Author details}

'The Kirby Institute, The University of New South Wales, Sydney, NSW, Australia. ${ }^{2}$ Division of HIV/AIDS and STI control, Hunan Provincial Centers for Disease Control and Prevention, Hunan, China. ${ }^{3}$ Comprehensive AIDS Research Center, Tsinghua University, Beijing, China.

Received: 20 August 2012 Accepted: 29 April 2013

Published: 7 May 2013

\section{References}

1. Guo W, Qu SQ, Ding ZW, et al: Situations and trends of HIV and syphilis infections among drug users in China, 1995-2009. Zhonghua Liu Xing Bing Xue Za Zhi 2010, 31(6):666-669.

2. Yao Y, Wang N, Chu J, et al: Sexual behavior and risks for HIV infection and transmission among male injecting drug users in Yunnan, China. Int J Infect Dis 2009, 13(2):154-161.

3. Li JR, Gong RY, Tian KL, et al: Study on the blood-borne virus co-infection and T lymphocyte subset among intravenous drug users. World J Gastroenterol 2007, 13(16):2357-2362.

4. Li JR, Gong RY, Li YY, et al: Research on HIV/Toxoplasma gondii co-infection and cytokine levels among intravenous drug users. Parasite Immunol 2010, 32(2):161-164.

5. Ruxrungtham K, Brown T, Phanuphak P: HIV/AIDS in Asia. Lancet 2004 364(9428):69-82

6. Weniger BG, Limpakarnjanarat $K$, Ungchusak $K$, et al: The epidemiology of HIV infection and AIDS in Thailand. AIDS 1991, 5(Suppl 2):S71-85.

7. Ministry of Health of the People's Republic of China: Joint United Nations Programme on HIV/AIDS, World Health Organization: 2009 Estimates for the HIV/AIDS Epidemic in China. Beijing: Ministry of Health of the People's Republic of China; 2010.

8. State Council AIDS Working Committee Office (SCAWCO): China 2010 UNGASS Country Progress Report (2008-2009). Beijing: Ministry of Health of the People's Republic of China; 2010.

9. Zheng LQ: Needle and syringe program sites have been increased to 1023 sites; 2012. http://211.154.163.181/jkb/html/2010-12/28/content_89385.htm.

10. Hammett TM, Bartlett NA, Chen Y, et al: Law enforcement influences on HIV prevention for injection drug users: Observations from a cross-border project in China and Vietnam. Int J Drug Policy 2005, 16(4):235-245.

11. Cohen JE, Amon JJ: Health and human rights concerns of drug users in detention in Guangxi Province, China. PLoS Med 2008, 5(12):e234.
12. State Council of the People's Republic of China: State council notice on strengthening HIV/AIDS prevention and control. State Council Document 2004 (7). Beijing: State Council; 2004

13. Sullivan SG, Wu Z: Rapid scale up of harm reduction in China. Int J Drug Policy 2007, 18(2):118-128.

14. Editor: Registered drug users exceeds 1.4 million in China. Chinese Journal of Drug Abuse Prevention and Treatment. 2011, 17(1):60.

15. Kwon JA, Iversen J, Maher $L$, et al: The impact of needle and syringe programs on HIV and HCV transmissions in injecting drug users in Australia: a model-based analysis. J Acquir Immune Defic Syndr 2009, 51(4):462-469.

16. Vickerman P, Kumaranayake L, Balakireva O, et al: The cost-effectiveness of expanding harm reduction activities for injecting drug users in Odessa, Ukraine. Sex Transm Dis 2006, 33(10 Suppl):S89-102.

17. Jenkins C, Rahman H, Saidel T, et al: Measuring the impact of needle exchange programs among injecting drug users through the National Behavioural Surveillance in Bangladesh. AIDS Educ Prev 2001, 13(5):452-461.

18. Wodak A, Cooney A: Do needle syringe programs reduce HIV infection among injecting drug users: a comprehensive review of the international evidence. Subst Use Misuse 2006, 41(6-7):777-813.

19. Bastos Fl, Strathdee SA: Evaluating effectiveness of syringe exchange programmes: current issues and future prospects. Soc Sci Med 2000, 51(12):1771-1782.

20. Wodak A: Lessons from the first international review of the evidence for needle syringe programs: the band still plays on. Subst Use Misuse 2006, 41(6-7):837-839.

21. Liu B, Sullivan SG, Wu Z: An evaluation of needle exchange programmes in China. AIDS 2007, 21(Suppl 8):S123-128.

22. Wu Z, Luo W, Sullivan SG, et al: Evaluation of a needle social marketing strategy to control HIV among injecting drug users in China. AIDS 2007, 21(Suppl 8):S115-122.

23. Lin P, Fan ZF, Yang F, et al: [Evaluation of a pilot study on needle and syringe exchange program among injecting drug users in a community in Guangdong, China]. Zhonghua Yu Fang Yi Xue Za Zhi 2004, 38(5):305-308.

24. Fang $Y X$, Wang $Y B$, Shi J, et al: Recent trends in drug abuse in China. Acta Pharmacol Sin 2006, 27(2):140-144.

25. Guydish J, Bucardo J, Young $M$, et al: Evaluating needle exchange: are there negative effects? AIDS 1993, 7(6):871-876.

26. Hartgers C, Buning EC, van Santen GW, et al: The impact of the needle and syringe-exchange programme in Amsterdam on injecting risk behaviour. AIDS 1989, 3(9):571-576.

27. van Ameijden EJ, Coutinho RA: Large decline in injecting drug use in Amsterdam, 1986-1998: explanatory mechanisms and determinants of injecting transitions. J Epidemio/ Community Health 2001, 55(5):356-363.

28. Junge $B$, Valente $T$, Latkin $C$, et al: Syringe exchange not associated with social network formation: results from Baltimore. AIDS 2000, 14(4):423-426.

29. Bluthenthal RN, Gogineni A, Longshore D, et al: Factors associated with readiness to change drug use among needle-exchange users. Drug Alcohol Depend 2001, 62(3):225-230.

30. Hurley SF, Jolley DJ, Kaldor JM: Effectiveness of needle-exchange programmes for prevention of HIV infection. Lancet 1997, 349(9068):1797-1800.

31. Zhou Y, Li X: Demographic characteristics and illegal drug use patterns among attendees of drug cessation programs in China. Subst Use Misuse 1999, 34(6):907-920.

32. United Nations Drug Control Program: China country profile. Bangkok: Regional Centre for East Asia and the Pacific; 2000.

33. The Joint United Nations Programme on HIV/AIDS, The United Nations International Drug Control Program: Drug use and HIV vulnerability: Policy research study in Asia. Bangkok: Regional Centre for East Asia and the Pacific; 2000.

34. Liu H, Grusky O, Zhu Y, et al: Do drug users in China who frequently receive detoxification treatment change their risky drug use practices and sexual behavior? Drug Alcohol Depend 2006, 84(1):114-121.

35. Marsch LA: The efficacy of methadone maintenance interventions in reducing illicit opiate use, HIV risk behavior and criminality: a metaanalysis. Addiction 1998, 93(4):515-532.

36. Corsi KF, Lehman WK, Booth RE: The effect of methadone maintenance on positive outcomes for opiate injection drug users. J Subst Abuse Treat 2009, 37(2):120-126.

37. Cohen DA, Wu SY, Farley TA: Comparing the cost-effectiveness of HIV prevention interventions. J Acquir Immune Defic Syndr 2004, 37(3):1404-1414. 
38. Laufer FN: Cost-effectiveness of syringe exchange as an HIV prevention strategy. J Acquir Immune Defic Syndr 2001, 28(3):273-278.

39. Harris ZK: Efficient allocation of resources to prevent HIV infection among injection drug users: the Prevention Point Philadelphia (PPP) needle exchange program. Health Econ 2006, 15(2):147-158.

40. Wilson DP, Kwon A, Anderson J, et al: Return on investment 2: Evaluating the cost-effectiveness of needle and syringe programs in Australia. Canberra, Australia: Publications Production Unit (Public Affairs, Parliamentary and Access Branch) Commonwealth Department of Health and Ageing; 2009 .

41. Zhang L, Yap L, Xun Z, et al: Needle and syringe programs in Yunnan. China yield health and financial return. BMC Public Health. 2011, 11:250.

42. Hammett TM, Wu Z, Duc TT, et al: 'Social evils' and harm reduction: the evolving policy environment for human immunodeficiency virus prevention among injection drug users in China and Vietnam. Addiction 2008, 103(1):137-145.

43. Gibson DR, Brand R, Anderson K, et al: Two- to sixfold decreased odds of HIV risk behavior associated with use of syringe exchange. J Acquir Immune Defic Syndr 2002, 31(2):237-242.

doi:10.1186/1477-7517-10-8

Cite this article as: Zhang et al:: Ability to access community-based

needle-syringe programs and injecting behaviors among drug users: a cross-sectional study in Hunan Province, China. Harm Reduction Journal 2013 10:8.

\section{Submit your next manuscript to BioMed Central and take full advantage of:}

- Convenient online submission

- Thorough peer review

- No space constraints or color figure charges

- Immediate publication on acceptance

- Inclusion in PubMed, CAS, Scopus and Google Scholar

- Research which is freely available for redistribution 